\title{
Application of Thin Metal Oxide Films in Acoustic Wave Chemical Sensors
}

\section{Esmeryan KD*}

Georgi Nadjakov Institute of Solid State Physics, Bulgaria

"Corresponding author: Esmeryan KD, MD, Georgi Nadjakov Institute of Solid State Physics, 72, Tzarigradsko Chaussee Blvd, 1784 Sofia, Bulgaria, Tel: + 9014170809; E-mail: karekin_esmeryan@issp.bas.bg

Received date: March 12, 2015 Accepted date: March 19, 2015 Published date: March 30, 2015

Copyright: (C) 2015 Esmeryan KD. This is an open-access article distributed under the terms of the Creative Commons Attribution License, which permits unrestricted use, distribution, and reproduction in any medium, provided the original author and source are credited.

\begin{abstract}
Acoustic wave chemical sensors have several features such as high resolution and sensitivity, fast responserecovery time, superb overall stability and high dynamic range, which determine their appropriateness for gas analyte detection. The usability of acoustic sensors requires specific knowledge relating to the sensing mechanism, the properties of different acoustic wave modes and the criteria for selection of sensitive layers, involved in a wide range of gas-phase applications. This paper reports the most commonly used metal oxide film coated acoustic wave sensors, as well as their operation principle and practical application. Several advantages and disadvantages of each particular acoustic wave device are identified and the selectivity of the sensors is presented and discussed.
\end{abstract}

Keywords: Chemical sensors; Acoustic waves

\section{Introduction}

Acoustic wave chemical sensors (AWCS) have attracted considerable interest of the sensor community because of important features such as high resolution and sensitivity, fast response-recovery time, superb overall stability and high dynamic range [1-3]. They can be classified in three main categories: sensors utilizing surface, bulk and guided acoustic waves. These waves are mechanical vibrations excited in piezoelectric crystals, where the wave velocity of propagation matches the velocity of sound in solids, hence the name acoustic. The surface acoustic waves (SAWs) are generated by means of two interdigital transducers (IDT), which are interlocking combshaped metal electrode devices with a certain finger periodicity, situated on a piezoelectric substrate [4]. By applying a RF signal, whose frequency matches the IDT's synchronous frequency, the former is converted most efficiently into a mechanical vibration. Thus, for SAW sensors, the finger periodicity determines the electrical periodicity and with the choice of substrate gives the fundamental resonant frequency. For the case of guided waves an additional metal grating or thin (nm range) solid film is needed between the IDTs to prevent wave diffraction into the bulk and confine the energy close to the surface $[5,6]$. Depending on the energy trapping mechanism, the generated acoustic wave can be surface transverse wave (STW) or Love wave (LW). STW and LW sensors are very similar to the SAW ones, but the choice of different crystal cut yields shear horizontal instead of Rayleigh wave. The third category of devices exploits bulk acoustic waves (BAWs). Their excitation is through an AT-cut quartz substrate mounted between two circular metal electrodes [7] or sandwich-type structure consisting of thin piezoelectric membrane (usually AlN or $\mathrm{ZnO}$ ) and two metallic layer electrodes [8]. Since AW sensors are fabricated on piezoelectric substrates, the detection process involves mechanical and/or electrical perturbations. Therefore, these devices are sensitive mainly to physical parameters, which may interact with the mechanical and/or electrical component of the wave.

This paper considers the operation principle of the most commonly used metal oxide film coated acoustic sensors. Several advantages and disadvantages of each device are identified. Finally, the selectivity of the sensors and their practical application are presented and discussed.

\section{Operation principle of metal oxide film coated AWCS}

\section{Acoustic sensors utilizing SAWs and STWs}

Regardless the chosen acoustic wave mode (Rayleigh SAW or STW) and device configuration (delay line or resonator), the operation principle of metal oxide film coated SAW sensors is identical and schematically illustrated on Figure 1.

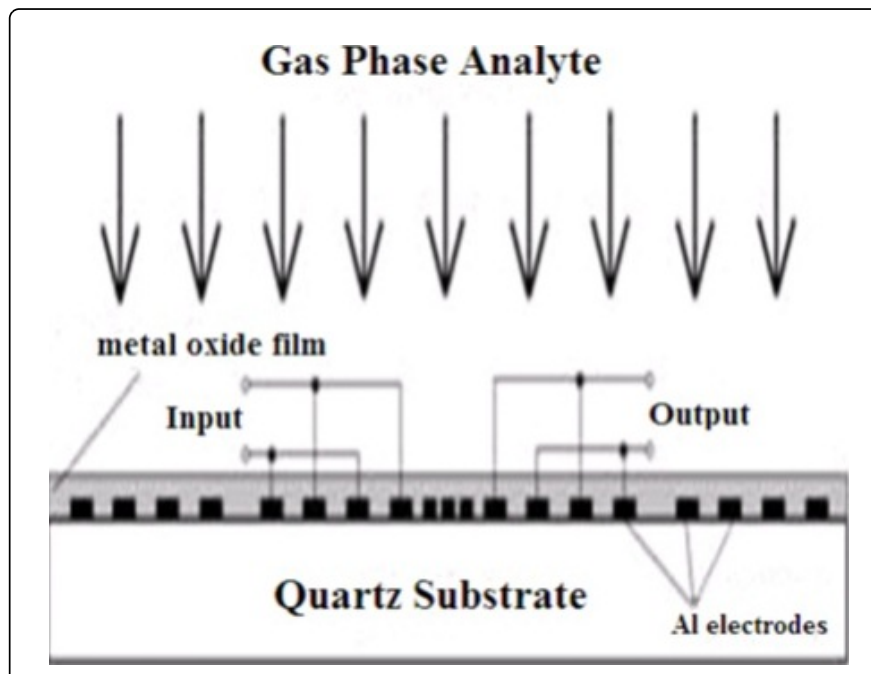

Figure 1: Operation principle of metal oxide film coated AWCS utilizing SAWs and STWs.

A delay line configuration or two-port resonator (shown on Figure 1) fabricated on a temperature compensated cut of piezoelectric quartz, typically ST-cut for SAW and AT-cut for STW, is coated with a thin $(\sim \mathrm{nm})$ metal oxide layer and mounted in a sensor head [9]. This type of sensitive coating is characterized by physical sorption, which 
Page 2 of 3

means that it has the ability to absorb/adsorb gas molecules if a chemical compound of interest is applied to its surface. Subsequently, when the gas is flushed away with dry air from the sensor head, all analyte molecules will restlessly escape from the film. During the gas sorption, the sensitive layer becomes heavier, leading to an increased mass loading on the sensor surface. As a result, the acoustic wave propagation velocity decreases and a mass dependent resonance frequency downshift $\Delta \mathrm{f}$, referred to as "sensor signal", is observed [10].

It has recently been reported, that metal oxide film coated acoustic sensors utilizing SAWs find wide application for high sensitive detection of volatile organic compounds [11], ammonia [12], hydrogen [13], nitrogen dioxide [14] etc. For instance, organic vapor concentrations in the range of $500-5000 \mathrm{ppm}$ result in a frequency downshift of $100 \mathrm{kHz}$ to $2.4 \mathrm{MHz}$ [11]. This is of crucial importance for monitoring the concentration of these gases in industrial areas and factories, as they can cause serious health problems. The major advantage of SAW devices is their high sensitivity and resolution due to the high operation frequency (up to the $\mathrm{GHz}$ range). However, they possess low temperature stability $[15,16]$, compared to the quartz crystal microbalance (QCM), which can be a serious drawback during measurements in "real world" conditions.

\section{Acoustic sensors utilizing BAWs}

The most commonly used BAW sensor is the QCM, developed by Sauerbrey in 1959 [7]. This device can operate well both in gas and liquid medium $[17,18]$ and its operation principle as a gas sensor is presented on Figure 2.

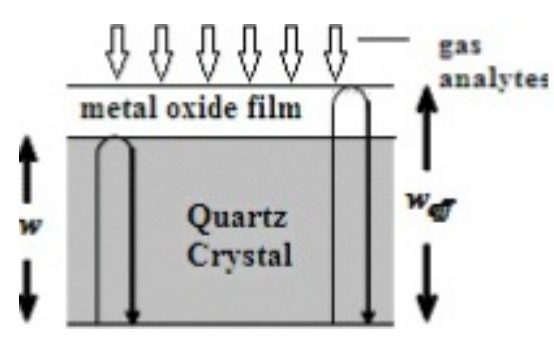

Figure 2: Operation principle of a metal oxide film coated QCM in a gas medium

The QCM consist of an AT-cut quartz crystal, sandwiched between two circular metal electrodes, and a metal oxide sensitive layer deposited on the upper surface, as shown in Figure 2. A simple pictorial acoustic view of an unloaded QCM shows that a strong reflection occurs at the solid-air interface [19] due to a major distinction in the acoustic impedances (Vacoustic wave/air $\sim 300 \mathrm{~m} / \mathrm{s}$; Vacoustic wave/solid $\sim 3000 \mathrm{~m} / \mathrm{s}$ ). In this case the crystal substrate forms an acoustic cavity in which a standing wave is created. The deposition of a metal oxide coating does not alter the fact that there is a strong reflection boundary at the solid-air interface, but leads to an extension of the cavity and decrease in the resonance frequency. By using the density of the layer this can be interpreted as a frequency downshift proportional to the mass per unit area i.e. Sauerbrey equation [7]. During gas sorption, the sensitive film changes its density and mass, which induces an additional frequency shift, known as "sensor signal", similarly to SAW sensors. The film bulk acoustic wave resonator (FBAR) has identical operation principle, but with a few advantages, including ability to fabricate devices using standard
CMOS processing and materials allowing integration with CMOS control circuitry; reduced volume and size, and higher sensitivity [8].

Metal oxide film coated QCMs find practical application as detectors of warfare agents [20] and various gas analytes [21]. The low detection limit to dimethyl methylphosphonate (35 ppb) and other gases is of key importance for anti-terrorism purposes and health risk assessment in industrial areas and factories. The main advantage of the QCM, compared to SAW sensors, is the extreme thermal stability $(<1$ $\mathrm{ppm} /{ }^{\circ} \mathrm{C}$ ) [22], but the crystal thickness dependent operation frequency leads to a lower mechanical robustness at high frequencies.

\section{Acoustic sensors utilizing guided waves}

These acoustic devices operate with "guided" shear horizontal (SH) surface acoustic wave modes. To obtain a wave guiding effect it is necessary to apply an additional metal grating or thin solid film $(\sim \mathrm{nm})$ onto sensor's surface, acting as a waveguide, which traps the energy close to the surface. The scheme of this effect is illustrated on Figure 3.

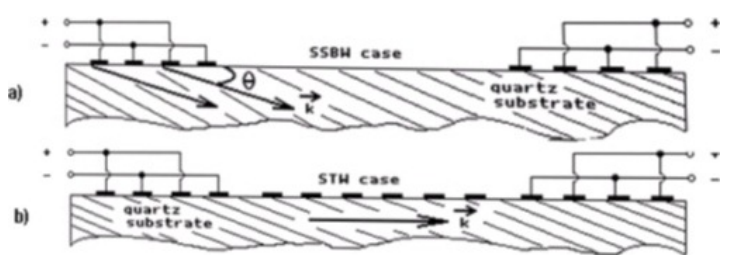

Figure 3: Schematic representation of the energy-trapping effect: a) surface shallow bulk wave (SSBW) and b) surface transverse wave (STW).

If the $\mathrm{SH}$-wave is excited and detected through two IDTs, separated by a free surface from each other as shown in Figure 3, then the wave is SSBW [23]. In this case, the wave energy is radiated under angle $\theta$ with respect to the surface and diffracts into the bulk of the crystal. This results in an increased insertion loss, especially if the IDTs are on a large distance from each other. However, if a metal strip grating, with an equal periodicity as the IDTs, is deposited between them, it slows down the wave because of the reflection in each individual metal strip. Thus, the k-vector becomes parallel to the surface, trapping the acoustic wave and preventing its diffraction into the bulk. Due to the confinement of most of the energy adjacent to the surface, these types of sensors are characterized by higher sensitivity compared to their QCM and SAW counterparts. By changing the metal strip grating with thin solid film, the wave can be transformed from STW to Love wave. The latter occurs only if the wave velocity in the layer is lower than in the substrate [6].

\section{Selectivity of metal oxide film coated AWCS}

Selectivity refers to the ability of a sensor to distinguish among the analyte under investigation and possible interferences [24]. For the case of acoustic wave chemical sensors, this property depends on the coating material. In "real world" conditions, perfect selectivity to a single chemical compound is unattainable if one uses simple polymer, organic or inorganic films (including metal oxides). However, desirable selectivity for a particular application could be achieved if the nature and the number of potential interferants are known [24]. The focus of research in the field of acoustic wave sensor development has been to achieve selectivity by using different types of coatings. The 
ideal coating will exhibit high sensitivity (maximum frequency deviation upon absorption/adsorption of a certain amount of gas) towards the analyte of interest, but low sensitivity to other species. One strategy to achieve this goal is by tailoring the chemical properties of the film to those of the target analyte. Hence, the sensitive layer will interact dominantly with the investigated chemical compound at the expense of the others in the mixture. Another possibility is to use an acoustic wave sensor array system [25], where $2 \times 2$ non-continuously working oscillators are equipped with coated SAW sensors. Although these strategies are powerful, the issue of selectivity continues to represent the most active area of research for this class of sensors [24].

\section{Conclusion}

The operation principle of the most commonly used metal oxide film coated AWCS was illustrated and discussed. The possible practical applications of these devices, as well as their advantages and disadvantages were identified. Finally, the problem with the selectivity of the sensors was presented and discussed.

\section{References}

1. Ballantine DS, Wohltjen H (1989) Surface acoustic wave devices for chemical analysis. Anal Chem 61: 704A-715A.

2. Hoummady M (1997) Acoustic wave sensors: design, sensing mechanisms and applications Smart Mater. Struct 6: 647-657.

3. Bender F, Wachter L, Voigt A, Rapp M (2003) Deposition of high quality coatings on SAW sensors using electrospray. Proceedings of the IEEE Sensors. Toronto, Canada.

4. White RM, Voltmer FW (1965) Direct piezoelectric coupling to surface elastic waves. Appl Phys Lett 7: 314-316.

5. Thompson DF, Auld BA (1986) Surface transverse wave propagation under metal strip gratings. Proc IEEE Ultrason Symp: 261-266.

6. Harding G, Du J (1997) Design and properties of quartz-based Love wave acoustic sensors incorporating silicon dioxide and PMMA guiding layers. Smart Mater Struct 6: 716-720.

7. Sauerbrey GZ (1959) Verwendung von Schwingquarzen zur Wagung dunner schichten und zur Mikrowagung Physics 155: 206-222.

8. Gabl R, Green E, Schreiter M, Primig R, et al (2003) Novel integrated FBAR sensors: A universal technology platform for bio and gas detection. Proc. IEEE Sens 2: 1184-1188.

9. Rapp M, Reibel J, Stier S, Voigt A, et al (1997) SAGAS: Gas Analyzing sensor systems based on surface acoustic wave devices-An issue of commercialization of SAW sensor technology. Proc IEEE Int Freq Cont Symp: 129-132.
10. Avramov ID (2011) Polymer coated Rayleigh SAW and STW resonators for gas sensor applications. Acoustic Waves - from Microdevices to Helioseismology 3: 521-545.

11. Tasaltin $\mathrm{C}$ (2012) Electrospun nanostructured $\mathrm{ZnO}$ thin films on SAW sensors for VOC detection. Int Meet Chem Sens : 1510-1512.

12. Bojadzhiev S, Georgieva V, Rassovska M (20100 Characterization of reactive sputtered $\mathrm{TiO} 2$ thin films for gas sensor applications. Jour of Phys Conf Ser 253: 1-6.

13. Huang FC1, Chen YY, Wu TT (2009) A room temperature surface acoustic wave hydrogen sensor with $\mathrm{Pt}$ coated $\mathrm{ZnO}$ nanorods. See comment in PubMed Commons below Nanotechnology 20: 065501.

14. Sadek AZ. A fast response $\mathrm{NO} 2$ gas sensor based on $\mathrm{ZnO}$ nanorod layered SAW transducer.

15. Esmeryan KD, Avramov ID, Radeva EI (2012) Temperature frequency characteristics of Hexamethyldisiloxane (HMDSO) polymer coated Rayleigh surface acoustic wave (SAW) resonators for gas-phase sensor applications Micromach. 3: 413-426.

16. Avramov ID, Esmeryan KD (2012) Thermal sensitivity of solid polymer coated surface transverse/Love wave based resonators on AT-cut quartz for sensor applications. Sens and Trans 147: 15-26.

17. Nomura T, Okuhara M (1982) Frequency shifts of piezoelectric quartz crystals immersed in organic liquids. Anal Chem 142: 281-284.

18. Schneider, Thomas W, Frye-Mason, Gregory C, Martin, et al (1998) Chemically selective coated quartz crystal microbalance array for detection of volatile organic chemicals. Proc SPIE 3539: 85-94.

19. McHale G, Roach P, Evans CR, Shirtcliffe NJ, et al (2008) Sensor response of superhydrophobic quartz crystal resonators. IEEE Freq Cont Symp: 698-704.

20. Pei Z, Ma X, Ding P, Zhang W, Luo Z, et al. (2010) Study of a QCM dimethyl methylphosphonate sensor based on a $\mathrm{ZnO}$-modified nanowire-structured manganese dioxide film. Sensors (Basel) 10: 8275-8290.

21. Vashist SK, Vashist P (2011) Recent advances in quartz crystal microbalance-based sensors. Jour Sens.

22. Bechmann R (1956) Frequency-temperature-angle characteristics of ATtype resonators made of natural and synthetic quartz Proc IRE 44: 1600-1607.

23. Avramov ID (1992) Microwave oscillators stabilized with surface transverse wave resonant devices IEEE Freq Cont Symp: 391-408.

24. Ballantine DS (1996) Performance Criteria: Selectivity in Acoustic Wave Sensors: Theory, Design and Physico-Chemical Applications: 239-241.

25. Chiang M-C, Hao HC, Chen-Yun H, Szu-Chieh L, et al. (2012) Gas sensor array based on surface acoustic wave devices for rapid multidetection. IEEE Conf Nanotech Mater Devices: 139-142. 